\title{
Time-Resolved Transient Grating Spectroscopy for Studies of Nonequilibrium Carrier Dynamics in Wide Band-Gap Semiconductors
}

\author{
K. JarašIūnas*, T. Malinauskas, K. Neimontas, V. Gudelis
}

AND R. Aleksiejūnas

Department of Semiconductor Optoelectronics

Institute of Materials Science and Applied Research

Vilnius University, Sauletekio Ave. 9-III, 10222 Vilnius, Lithuania

Using interdisciplinary fields relevant to a highly excited semiconductor - nonequilibrium phenomena in high density plasma, light-induced changes of optical properties, and dynamic holography, we developed time-resolved four-wave mixing technique for monitoring the spatial and temporal carrier dynamics in wide band-gap semiconductors. This opened a new possibility to analyse fast electronic processes in a non-destructive "all-optical" way, i.e. without any electrical contacts. This technique allowed evaluation of recombination and transport processes and the determination of important carrier parameters which directly reveal the material quality: carrier lifetime, bipolar diffusion coefficients, surface recombination rate, nonlinear recombination rate, diffusion length, threshold of stimulated recombination. The recent experimental studies of differently grown group III-nitrides (heterostructures and free standing films) as well silicon carbide epilayers by nondegenerate picosecond four-wave mixing are presented.

PACS numbers: 72.20.Jv, 78.47.+p, 78.55.Cr

\section{Introduction}

The development of advanced wide band-gap semiconductor materials requires novel tools in order to characterize their physical properties. Direct relationship between nonresonant, light induced refractive index modulation $\Delta n(x, t)$

*corresponding author; e-mail: kestutis.jarasiunas@ff.vu.lt 
by nonequilibrium carriers $\Delta N(x, t)$ and holographic approach for monitoring dynamics of $\Delta n$ allowed development of innovative holographic measurement technique - the time-resolved (TR) four-wave mixing (FWM) on free carrier gratings. Recently TR FWM under picosecond excitation was applied for study of competition between nonradiative recombination at dislocations and radiative interband processes in GaN layers with varying dislocation density [1], determination of bimolecular recombination coefficient and its temperature dependence [2] in hydride vapor phase epitaxy grown (HVPE-grown) free standing films, control of stimulated emission threshold in lasing compounds [3], carrier localization effects in InGaN alloys by direct measurements of In-content dependent diffusion coefficient values and carrier lifetimes values [4], recombination activity of iron-related traps and dislocations in highly resistive GaN layers [5]. In epitaxial SiC, origin of fast decay transients of bipolar nonequilibrium carrier plasma was analysed [6]. In this paper, we briefly review the metrological potential of TR FWM for investigation of carrier-density, defect-density, and temperature dependent nonequilibrium carrier dynamics in differently grown GaN layers, InGaN alloys, and $\mathrm{SiC}$ crystals.

\section{Basic principles of four-wave mixing technique}

Light diffraction on light-induced dynamic gratings has been an object of intensive studies a decade ago (see review papers [7-9]). A particular advantage of this technique is its capability to monitor carrier generation, transport and recombination, as it bridges the photoelectrical properties of semiconductors with related optical nonlinearities, which are monitored by time-resolved dynamic holography. The latter approach - excitation of a crystal with light interference pattern has number of advantages against the other "excite-probe" techniques (e.g. induced absorption, reflection, refraction, or Z-scan). Light diffraction creates new beams in a dark field, thus increasing signal-to-noise ratio. Next, the intensity of diffracted beams nonlinearly reflects the modulation amplitude of refractive index. In addition, spatial modulation of carrier density allows simple monitoring of diffusion processes along the grating vector by varying the grating period, and, thus, enables separation of diffusion from recombination. Finally, variation of excitation wavelength leads to various thicknesses of photoexcited region. The spatial in-depth resolution is defined by thickness of a bulk crystal, if excited by a quantum with energy $h \nu<E_{\mathrm{g}}$, and may reach a sub-micron thickness at strong light absorption $\left(h \nu>E_{\mathrm{g}}\right)$. In-plane resolution is limited by number of grating lines in the excited spot and varies from $50 \mu \mathrm{m}$ to $1-2 \mathrm{~mm}$.

The FWM technique uses the quantitative relationships in order to bridge the measured diffraction characteristics with light-induced modulation of optical properties and with the optical and electrical parameters of a semiconductor. Therefore, a specific knowledge of dynamic holography, nonlinear optics, and semiconductor physics is required. Dynamic holography provides optical configurations to create and monitor the spatial refractive index modulation $n(x, t)=n_{0}+\Delta n(t) \cos (K x)$ 
as well relationship between the diffraction efficiency of a grating $\eta=(\pi \Delta n d / \lambda)^{2}$, while the nonlinear optics gives quantitative ratios between the modulation mechanisms of optical properties and the modulation amplitude $\Delta n$. In the given case, the refractive index change at $1064 \mathrm{~nm}$ (well below the band gap) is determined by free carrier density $N$ according to Drude-Lorentz model: $\Delta n=-n_{\mathrm{eh}} \Delta N$, where the coefficient $n_{\mathrm{eh}}$ is the refractive index change by one electron-hole pair $n_{\mathrm{eh}}[3]$ and equals to $\approx 1 \times 10^{-21} \mathrm{~cm}^{-3}$ for GaN at the wavelength of the probe beam. This coefficient together with the thickness of excited region $d$ determines the sensitivity of the FWM technique, i.e. the minimum value of product $(\Delta n d)$, which can be detected in diffraction. Usually, the signal/noise ratio $\approx 1$ is reached at diffraction efficiency of $\eta \cong 10^{-5}$, which corresponds to $(\Delta n d) \geq 0.001$, and this limit corresponds to the lowest carrier density of $10^{14}-10^{15} \mathrm{~cm}^{-3}$ (in a few $\mathrm{mm}$ thick bulk crystals) or $10^{18} \mathrm{~cm}^{-3}$ (in a few $\mu \mathrm{m}$ thick epitaxial layer) which can be assessed by the FWM technique.

From the point of view of materials research, the most important are the material equations, which join quantitatively the generation, recombination, and transport processes with the measured characteristics of FWM, i.e. the grating kinetics $\eta=f(t)$ at various periods $\Lambda$ and the exposure characteristics (dependence of diffraction efficiency on excitation intensity $\eta=f\left(I_{0}\right)$ ). At last but not least, the semiconductor growth technologies provide variety of materials covering heteroand homostructures, highly conductive and semi-insulating bulk crystals, thin films and multiple quantum well structures for investigation of carrier dynamics, which is the most important characteristics of technologically advanced materials.

\section{Experimental setup}

Carrier dynamics in $\mathrm{GaN}$ and $\mathrm{SiC}$ structures was investigated in wide excitation and temperature range by using the non-degenerate picosecond FWM setup (Fig. 1). An interference pattern of two 25 ps duration laser beams at $355 \mathrm{~nm}$ wavelength $(h \nu=3.49 \mathrm{eV})$ or $266 \mathrm{~nm}(h \nu=4.43 \mathrm{eV})$ was strongly absorbed in a sample and created a modulated free-carriers distribution $N(x)=N_{0}[1+\cos (2 \pi x / \lambda)]$ with period $\Lambda$, varying in range from $\approx 3$ to $14 \mu \mathrm{m}$. In turn, the refractive index of a material was temporarily changed and created a dynamic diffraction grating. The grating was probed by a weakly absorbed beam at $1064 \mathrm{~nm}$ which propagated through the sample and created diffracted beams behind the sample. The exciting light at $355 \mathrm{~nm}$ was absorbed in $\alpha^{-1} \approx 100 \mathrm{~nm}$ thin surface layer of GaN or $50 \mu \mathrm{m}$ thick layer of $\mathrm{SiC}$, creating the bipolar carrier plasma with density in range $10^{19}$ or $10^{18} \mathrm{~cm}^{-3}$, correspondingly. Carrier diffusion from surface to the bulk increased the excited layer thickness up to 1-2 $\mu \mathrm{m}$ (for $\mathrm{GaN}$ ) in time domain of 2 nanoseconds, which corresponds to the probe-beam delay time in the measurements. The grating decay kinetics were measured by performing 20-40 measurements at a fixed probe beam delay time $\Delta t$ and integrating the data in a required intensity window of $I_{0} \pm \Delta I_{0}$ (usually $\Delta I_{0} / I_{0}<5 \%$ ). Exposure characteristics of FWM 


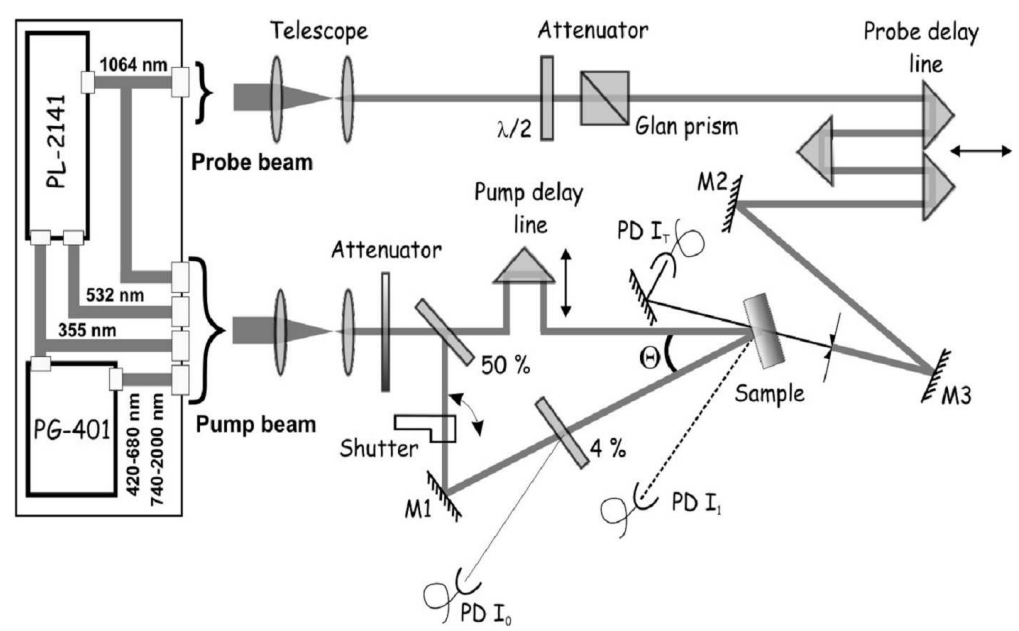

Fig. 1. Experimental setup of TR picosecond FWM. The transient grating is recorded by light interference field at a selected UV wavelength (355 $\mathrm{nm}$ or $266 \mathrm{~nm}$ ), while the grating decay is probed by the delayed IR beam at $1064 \mathrm{~nm}$. The energies of recording beam $I_{0}$, transmitted probe beam $I_{\mathrm{T}}$, and its diffracted part $I_{1}$ are controlled by silicon photodetectors PD and data acquisition system.

were measured by collecting 500-1000 laser shots at different excitation energies. The data acquisition system via the programmable Lab-view software monitors the experiment, processes the data, and presents the measured FWM characteristic in real time.

\section{Samples}

The experiments were carried on a set of differently grown GaN samples: by standard metalorganic chemical vapor deposition (MOCVD) layers, by epitaxial lateral overgrowth (ELO) under specific surface treatment (formation of 20-40 nm size randomly distributed GaN islands in the amorphous SiN layer, thereby leading to a maskless ELO process) [10], as well on 300-400 $\mu \mathrm{m}$ thick free-standing layers, grown by HVPE [11]. The set with threading dislocation (TD) density in range from $\approx 10^{6}$ to $10^{10} \mathrm{~cm}^{-2}$ provided possibility to study carrier recombination rate governed by dislocations as nonradiative recombination centers and bimolecular recombination processes in less defected HVPE layers. $\operatorname{In}_{x} \mathrm{Ga}_{1-x} \mathrm{~N}$ heterostructures with varying In-content have been studied in order to reveal role of carrier localization effect to carrier diffusion and recombination at high excitation. The $35 \mu \mathrm{m}$ thick $n$-type $4 \mathrm{H}$-SiC epilayers, grown by CVD on heavily nitrogen doped $4 \mathrm{H}-\mathrm{SiC}$ substrates [12] have been studied in very wide excitation range to get deeper insight into origin of nanosecond FWM relaxation times. 


\section{Results and discussions}

Two-dimensional continuity equation has been employed for modeling of spatial and temporal carrier redistribution $N(x, z, t)$ after their generation by a short laser pulse

$$
\begin{aligned}
& \frac{\partial N(x, z, t)}{\partial t}=G(x, z, t)+\nabla[D(N) \nabla N(x, z, t)]-\frac{N(x, z, t)}{\tau_{\mathrm{R}}} \\
& \quad-B N^{2}(x, z, t)-C N^{3}(x, z, t) .
\end{aligned}
$$

The model takes into account both linear and nonlinear carrier recombination; the latter was governed by quadratic recombination rate $1 / \tau_{\mathrm{RB}}=B N$ with bimolecular recombination coefficient $B$ as well by the cubic (Auger) recombination rate $1 / \tau_{\mathrm{RC}}=C N^{2}$ with the Auger recombination coefficient $C$. The bipolar diffusion with carrier-density dependent coefficient $D(N)$ in high density plasma was also taken into account. Equation (1) describes instantaneous carrier distribution along the grating vector $(x)$ and into the depth $(z)$, if the sample thickness is larger than the carrier diffusion length (e.g. in HVPE grown GaN, or in $\mathrm{SiC}$ at $266 \mathrm{~nm}$ excitation). This approach is essential for semi-infinite media as the probe beam propagates through the whole structure (active layer, interlayer, and substrate) and integrates the plasma modulation over the sample depth, thus making the diffraction signal dependent on the total number of photogenerated electron-hole pairs in the sample but not on a specific density profile. We note that the latter feature makes the FWM technique more advantageous for time-resolved studies of carrier dynamics than the well-known photoluminescence (PL) technique under similar excitation conditions [13].

Experimentally, the measured grating decay kinetics at various grating periods provided the bipolar diffusion $D$ and carrier lifetime $\tau_{\mathrm{R}}$ values according to the relationship $1 / \tau_{\mathrm{G}}=1 / \tau_{\mathrm{R}}+1 / \tau_{\mathrm{D}}$, where $\tau_{\mathrm{D}}=\Lambda^{2} / 4 \pi^{2} D$ is the diffusive grating decay time. Table summarizes the photoelectric parameters of differently grown GaN layers. It shows the range of bipolar diffusion length $L_{\mathrm{D}}$ varying from 0.1 to $1 \mu \mathrm{m}$, carrier lifetimes varying in two orders of magnitude (from 60 ps to $5.3 \mathrm{~ns}$ ), and the bipolar diffusion coefficients being less dependent on dislocation density. To enhance contribution of bimolecular recombination in less dislocated samples, the measurements have been performed at lower temperatures, as the bimolecular recombination coefficient $B$ is temperature dependent $\left(B \propto T^{-3 / 2}[14]\right)$. In addition, the instantaneous nonlinear recombination rate $\tau_{\mathrm{R}}(N)$ is influenced by carrier diffusion to the depth, as $D$ is also temperature dependent. Numerical simulation of spatial and temporal carrier dynamics, using the determined $D(T)$ values, was used to fit the experimental data and extract the coefficient $B$ at various temperatures. In this way, the numerical fitting of measured FWM kinetics provided the $B(T)$ dependence in 10-300 $\mathrm{K}$ range and corresponding values of $B=2 \times 10^{-11} \mathrm{~cm}^{3} / \mathrm{s}$ at $300 \mathrm{~K}$ and $B=2 \times 10^{-9} \mathrm{~cm}^{3} / \mathrm{s}$ at $10 \mathrm{~K}[1]$. 
TABLE

Dislocation-density dependent photoelectric parameters of GaN layers.

\begin{tabular}{c|c|c|c|c|c}
\hline \hline $\begin{array}{c}\text { Growth } \\
\text { technology }\end{array}$ & $\begin{array}{c}\text { Thickness } \\
{[\mu \mathrm{m}]}\end{array}$ & $\begin{array}{c}\text { Dislocation } \\
\text { density }\left[\mathrm{cm}^{-2}\right]\end{array}$ & $\begin{array}{c}\tau_{\mathrm{R}} \\
{[\mathrm{ns}]}\end{array}$ & $\begin{array}{c}D_{\mathrm{a}} \\
{\left[\mathrm{cm}^{2} / \mathrm{s}\right]}\end{array}$ & $\begin{array}{c}L_{\mathrm{D}} \\
{[\mu \mathrm{m}]}\end{array}$ \\
\hline $\mathrm{MOCVD} / \mathrm{Al}_{2} \mathrm{O}_{3}$ & 3 & $10^{10}$ & 0.1 & 1.7 & 0.1 \\
$\mathrm{MOCVD} / \mathrm{Al}_{2} \mathrm{O}_{3}$ & 2.6 & $10^{9}$ & 0.7 & 1.7 & 0.34 \\
$\mathrm{MOCVD} / \mathrm{Al}_{2} \mathrm{O}_{3}$ & $3-4$ & $5 \times 10^{8}$ & $0.9-1.1$ & $1.7-1.8$ & 0.42 \\
$\mathrm{Micro}-\mathrm{ELO}$ & 10 & $5 \times 10^{7}$ & 2.7 & 2.4 & 0.8 \\
$\mathrm{HVPE}$ & 270 & $2.5 \times 10^{7}$ & 2.9 & $1.9-2.9$ & $0.7-0.9$ \\
$\mathrm{HVPE}$ & 400 & $5 \times 10^{6}$ & $3.3-5.2$ & $1.9-2.7$ & $0.94-1$
\end{tabular}

At excitation of InGaN/GaN heterostructures, the main part of photoexcited carriers is confined in the front layer, since the InGaN/GaN interface presents potential barriers both for electrons and holes. Carrier localization effects, dependent on In content, were studied by measuring the kinetics of gratings with different periods (from 3 to $10.5 \mu \mathrm{m}$ ). The decrease in $D$ value from 1.5 to $0.9 \mathrm{~cm}^{2} / \mathrm{s}$ with increasing the In content (from 8 to 15\%) was found (Fig. 2) and attributed

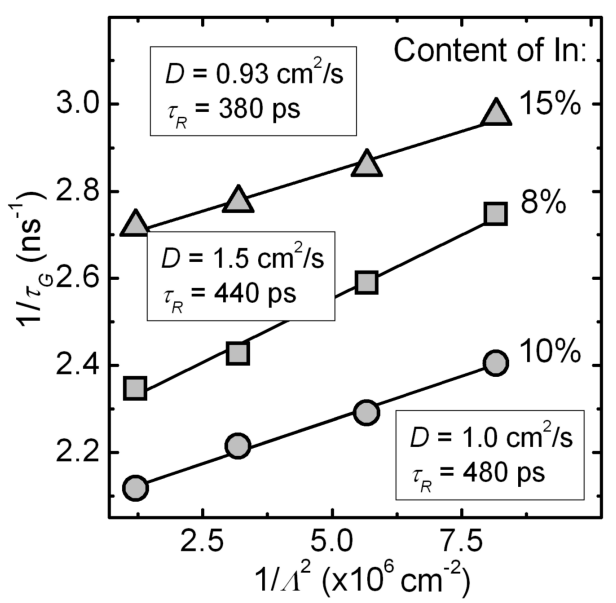

Fig. 2. Nonequilibrium carrier parameters in InGaN/GaN heterostructures with different In content, determined from FWM kinetics at various grating periods.

to higher concentration of localized states as well as to presence of the potential barriers in the vicinity of the In inclusions [15]. Partial screening of the barriers at even higher excitations resulted in a slightly higher value of $D$ (e.g. it was found equal to $\approx 2.1 \mathrm{~cm}^{2} / \mathrm{s}$ for $x=8 \%$ ). The correlation of carrier lifetime with In content (Fig. 2) was found more complicated, as the $\tau_{\mathrm{R}}$ value is governed by the localized, the "free" carriers, and by contribution of nonlinear recombination. The FWM kinetics provided the average recombination rate of all simultaneously 
contributing mechanisms. With increasing In content from 8 to $10 \%$, the $\tau_{\mathrm{R}}$ value increased due to carrier localization, while at $15 \%$ of In the recombination rate became faster (due to essential decrease in microcrystalline quality at In content above $10 \%$ [16]). In the latter case, the threading dislocations reach the In-rich local areas at the epilayer surface, and dominate in recombination. Therefore FWM technique allowed simple monitoring of an optimal In content to localize carriers, but not to cut down the carrier lifetime, which is required to reach the threshold of stimulated recombination.

Optical carrier injection in SiC epilayers at two different wavelengths (355 or $266 \mathrm{~nm}$ ) with quantum energies above the band-gap $E_{\mathrm{g}}$ made it possible to vary in-depth resolution, reach very high nonequilibrium carrier density, and study carrier-density dependent photoelectric processes. The excited region thickness varied from $d_{\text {eff }} \sim \alpha^{-1} \approx 50 \mu \mathrm{m}$ to $1-2 \mu \mathrm{m}$, and the photogenerated carrier density covered range from $2 \times 10^{16} \mathrm{~cm}^{-3}$ to $10^{19} \mathrm{~cm}^{-3}$. For carrier concentration above $\approx 3 \times 10^{18} \mathrm{~cm}^{-3}$, the decrease in carrier lifetime down to $\approx 2 \mathrm{~ns}$ with excitation was measured. Numerical modeling of recombination processes at high

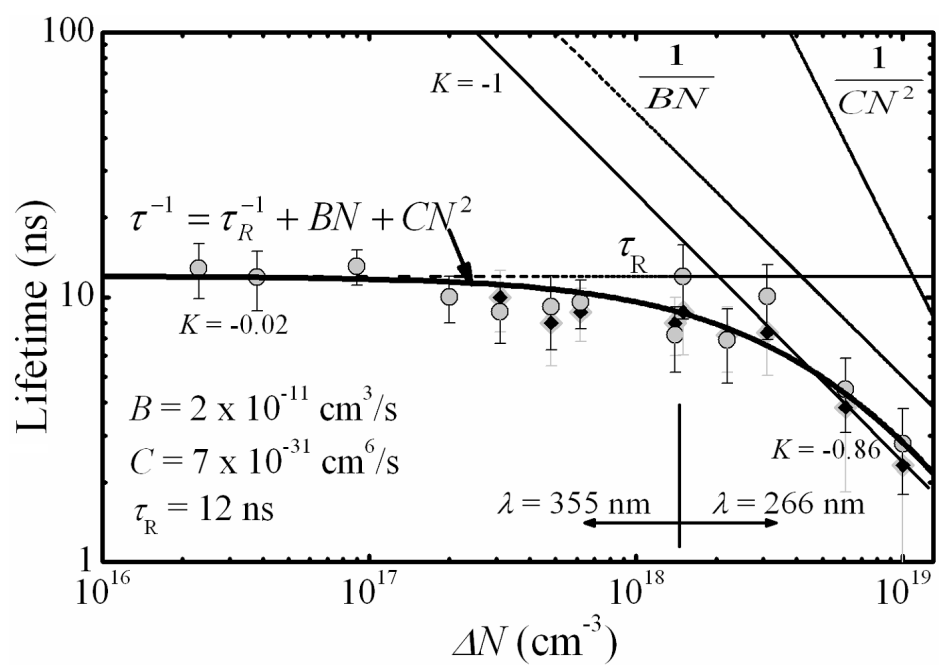

Fig. 3. Dependencies of carrier recombination time on nonequilibrium carrier density in $n$-type (circles) and nominally undoped (diamonds) 4H-SiC epilayers. Solid curve is the numerical fit. Dotted lines indicate the nonlinear recombination rates. The index $K$ stands for the slope value of the plotted dependence $\tau_{\mathrm{R}} \propto N^{K}$ in $\log$-log scale.

excitations allowed us to attribute the observed decrease in the carrier lifetime to the bimolecular (radiative) carrier recombination, as indicated by the solid curve in Fig. 3; the latter was simulated using Eq. (1) and the following parameters: linear carrier recombination time $\tau_{\mathrm{R}}=12 \mathrm{~ns}$, bimolecular recombination coefficient $B=$ $2 \times 10^{-11} \mathrm{~cm}^{3} / \mathrm{s}$, and Auger recombination coefficient $C=7 \times 10^{-31} \mathrm{~cm}^{6} / \mathrm{s}$. Dotted lines in the plot indicate the quantified recombination rates. In this way, more 
correct and higher value of recombination coefficient $B=(3 \pm 1) \times 10^{-11} \mathrm{~cm}^{3} / \mathrm{s}$ was derived in nominally undoped $4 \mathrm{H}-\mathrm{SiC}$ epilayers.

\section{Conclusions}

Time-resolved picosecond FWM has been proved a versatile technique to study photoelectric processes and determine by optical means the following parameters of nonequilibrium carriers in GaN, InGaN, and 4H-SiC crystals: carrier lifetimes in range from $10 \mathrm{ps}$ to $12 \mathrm{~ns}$, diffusion length in 0.1 to $1 \mu \mathrm{m}$ range, bipolar diffusion coefficients in range $0.9-2.9 \mathrm{~cm}^{2} / \mathrm{s}$, and bimolecular recombination rate $B=2 \times 10^{-11} \mathrm{~cm}^{3} / \mathrm{s}$ in $\mathrm{GaN}$ and $4 \mathrm{H}-\mathrm{SiC}$ at $300 \mathrm{~K}$. On the other hand, the techniques are complementary and can be used for more detailed studies of competition of radiative/nonradiative channels of carrier recombination as well for role of diffusion processes. The FWM technique has been recently implemented in a holographic module HOLO-2 by Ekspla Co. (www.ekspla.com), which has been used by Sensor Electronic Technology Inc. (USA) in the development of advanced III-nitride based materials for deep UV optoelectronic devices [17].

\section{Acknowledgments}

The work was supported by the European Commission Contract No. G5MACT-2002-04047. The research team at Vilnius University acknowledges cooperation links with Linköping University (Sweden), Lumilog Co. (France), and SET Inc. (USA), which provided numerous samples for joint studies.

\section{References}

[1] K. Jarašiūnas, T. Malinauskas, A. Kadys, S. Miasojedovas, S. Juršènas, A. Žukauskas, D. Gogova, A. Kakanakova, E. Janzen, H. Larsson, B. Monemar, B. Beaumont, P. Gibart, Phys. Status Solidi C 2, 1006 (2005).

[2] T. Malinauskas, K. Jarašiūnas, R. Aleksiejūnas, D. Gogova, B. Monemar, B. Beaumont, P. Gibart, Phys. Status Solidi B 7, 1426 (2006).

[3] S. Jursenas, S. Miasojedovas, G. Kurilcik, A. Zukauskas, R. Aleksiejunas, T. Malinauskas, M. Sudzius, K. Jarasiunas, Acta Phys. Pol. A 107, 240 (2005).

[4] K. Jarašiunas, R. Aleksiejūnas, M. Sūdžius, S. Miasojedovas, S. Juršènas, A. Žukauskas, M. Shur, E. Kuokstis, M. Asif Khan, Phys. Status Solidi A 202, $820(2005)$.

[5] Z. Bougrioua, M. Azize, P. Gibart, T. Malinauskas, K. Neimontas, A. Mekys, J. Storasta, K. Jarašiūnas, to be published.

[6] K. Neimontas, T. Malinauskas, R. Aleksiejūnas, M. Sūdžius, K. Jarašiūnas, L. Storasta, J.P. Bergman, E. Janzen, Semicond. Sci. Technol. 21, 952 (2006).

[7] H.J. Eichler, P. Gunter, D. Pohl, Light-Induced Dynamic Gratings, Springer Series in Optical Sciences, Vol. 50, Springer, Berlin 1986.

[8] R.K. Jain, M.B. Klein, in: Optical Phase Conjugation, Ed. R.A. Fisher, Acad. Press, New York 1983, Ch. 10. 
[9] Nonlinear Optics in Semiconductors, Vols. I \& II, Eds. E. Garmire, A. Kost, Semiconductors and Semimetals, Vol. 59, Acad. Press, New York 1999.

[10] E. Frayssinet, B. Beaumont, J.P. Faurie, Pierre Gibart, Zs. Makkai, B. Pécz, P. Lefebvre, P. Valvin, MRS Internet J. Nitride Semicond. Res. 7, 8 (2002).

[11] D. Gogova, A. Kasic, H. Larsson, C. Hemingsson, B. Monemar, F. Tuomisto, K. Saarinen, L. Dobos, B. Pecz, P. Gibart, B. Beaumont, J. Appl. Phys. 79, 799 (2004).

[12] A. Ellison, J. Zhang, A. Henry, E. Janzen, J. Crystal Growth 236, 225 (2002).

[13] T. Malinauskas, K. Jarašiūnas, Acta Phys. Pol. A 108, 781 (2005).

[14] A. Dmitriev, A. Oruzheinikov, J. Appl. Phys. 86, 3241 (1999).

[15] M. Ryu, E. Kuokstis, C. Chen, J. Yang, G. Simin, M. Khan, G. Sim, P. Yu, Solid State Commun. 126, 329 (2003).

[16] A. Ponce, S. Srinivasan, A. Bell, L. Geng, R. Liu, M. Stevens, J. Cai, H. Omiya, H. Marui, S. Tanaka, Phys. Status Solidi B 240, 273 (2003).

[17] R.S.Q. Fareed, J.P. Zhang, R. Gaska, G. Tamulaitis, J. Mickevicius, R. Aleksiejunas, M.S. Shur, M. Asif Khan, Phys. Status Solidi C 2, 2095 (2005). 